\title{
Evaluation of automated systems for aminoglycosides and fluoroquinolones susceptibility testing for Carbapenem- resistant Enterobacteriaceae
}

\author{
Zhichang Zhao ${ }^{1 \dagger}$, Fangjun Lan ${ }^{2 \dagger}$, Maobai Liu', Weiyuan Chen ${ }^{3}$, Liya Huang ${ }^{3}$, Qili Lin ${ }^{3}$ and Bin Li ${ }^{2 *}$
}

\begin{abstract}
Background: Automated systems (MicroScan WalkAway 96 Plus, Phoenix 100, and Vitek 2 Compact) are widely used in clinical laboratories nowadays. The aim of this study is to evaluate the performance of these three systems for susceptibility testing of aminoglycosides and fluoroquinolones against Carbapenem-resistant Enterobacteriaceae (CRE).

Methods: A total of 75 CRE isolates were used in this study. Quinolone resistance determinants (QRDs) (qnrA, anrB, anrC, $q n r D, a n r S, \operatorname{aac}\left(6^{\prime}\right)-1 b-c r, o q \times A B$ and $\left.q e p A\right)$ and aminoglycoside resistance determinants (ARDs) (aac( $\left.6^{\prime}\right)-1 b$, armA, $n p m A$, $r m t A, r m t B, r m t C, r m t D$ and $r m t E$ ) of these CRE were screened by PCR. The MICs of aminoglycosides (gentamicin and amikacin) and fluoroquinolones (ciprofloxacin and levofloxacin) to CRE obtained with the automated systems were compared with the reference method (agar dilution method).
\end{abstract}

Results: Totally, 97.3\% (73/75) of CRE harbored QRDs. The anr gene was the most common QRD determinant identified in 68 (96.7\%), followed by aac (6')-lb-cr in 56 (74.7\%), oqxAB in 23 (30.7\%), and qepA in 2 (2.7\%), respectively. 22.7\% (17/ 75) of CRE harbored ARD determinants. $r m t A, r m t B$ and $n p m A$ were identified among these isolates in $6(8.0 \%), 6(8.0 \%)$ and 5 (6.7\%), respectively. A total of 900 results were obtained in this study. Overall, the total error rate was $9.89 \%$. Twenty-eight very major errors (3.11\%), 22 major errors (2.44\%) and 39 minor errors (4.33\%) were identified against agar dilution method. The very major errors were almost evenly distributed between results for fluoroquinolones (2.89\%) and aminoglycosides (3.33\%), while the major errors and minor errors were more commonly found in the results of fluoroquinolones (3.11\% and 6.44\%, respectively) than aminoglycosides ( $1.78 \%$ and $2.22 \%$, respectively).

Conclusions: Our study shows that testing difficulties in susceptibility testing do exist in automated systems. We suggest clinical laboratories using automated systems should consider using a second, independent antimicrobial susceptibility testing method to validate aminoglycosides and fluoroquinolones susceptibility.

Keywords: Automated identification systems, Carbapenem-resistant Enterobacteriaceae, Aminoglycosides, Fluoroquinolones

\footnotetext{
* Correspondence: leonlee307@hotmail.com

${ }^{\dagger}$ Equal contributors

2Department of Clinical Laboratory, Fujian Medical University Union Hospital,

29 Xinquan Rd, Fuzhou, Fujian 350001, People's Republic of China

Full list of author information is available at the end of the article
} 


\section{Background}

Carbapenem-resistant Enterobacteriaceae (CRE) has spread throughout the world nowadays [1-3]. CRE usually show high levels of resistance to many types of antibiotics. Infections caused by CRE will pose a serious threat to patients for the limited therapeutic options. Therefore, CRE infections are always associated with poor outcomes and high mortality rate at the present time [4-6]. The most optimal treatment options for CRE infections have not been well defined. Current treatment options include the use of some older agents either in monotherapy or in combination therapy, such as aminoglycosides and fluoroquinolones [7, 8]. Aminoglycosides and fluoroquinolones are two different important types of antimicrobial agents for the treatment of life-threatening bacterial infections. Aminoglycosides have demonstrated in vitro activity against CRE, and are often used as part of combination regimens $[9,10]$. While fluoroquinolones are sometimes used to combat urinary tract infection caused by CRE [11].

The task of clinical laboratories and microbiologists to perform proper antimicrobial susceptibility test and interpretation constitutes an important initial step in implementing appropriate treatments and antibiotic use policies [12]. In this regard, the accuracy of different antimicrobial susceptibility testing methods may allow physicians to prescribe safe and effective antibiotics to treat infections. Automated systems have been used widely in many clinical laboratories for species identification of the pathogens and susceptibility testing. These systems could decrease the labor and save time compared to those for standardized methods. But probable errors reported by automated systems maybe have serious implications for the clinical outcome for patients with multiple drug resistant bacteria infections, such as CRE infections. Unfortunately, there is no information on the accuracy of these systems in detecting the susceptibility to aminoglycosides and fluoroquinolones with CRE currently.

In China, according to the CHINET report, the incidence of CRE has increased remarkably in the last 10 years [13]. In most Chinese hospitals, the susceptilbility of CRE to aminoglycosides and fluoroquinolones are detected using automated systems. Phoenix 100, Vitek-2 Compact and MicroScan WalkAway 96 Plus are the most common automated identification systems currently used in our country. The aim of the present study was to assess the performance of three automated instruments for the susceptibility testing of aminoglycosides and fluoroquinolones against CRE isolates.

\section{Methods}

\section{Clinical isolates}

A total of 75 carbapenem-resistant Enterobacteriaceae were included in this study. They were collected at
Fujian Medical University Union Hospital in Fuzhou, China, and some were reported previously [14].

\section{Antimicrobial susceptibility tests}

Aminoglycosides (gentamicin and amikacin) and fluoroquinolones (ciprofloxacin and levofloxacin) susceptibility tests were conducted simultaneously following the manufacturers' instructions using the three different automated systems: MicroScan WalkAway 96 Plus (SIEMENS AG FWB, Germany) with NC 50 cards, Phoenix 100 (Becton, Dickinson and Company, USA) with NMIC/ID-4 panels and Vitek-2 Compact (Bio Mérieux, France) with AST-GN16 cards. All the assays were accomplished in the research laboratory of Fujian Medical University Union Hospital, China. The susceptibility breakpoints of the three commercial systems were interpreted as recommended by the Clinical and Laboratory Standards Institute(CLSI) [15]. Reference MIC values for the tested isolates were determined by agar dilution method with Mueller-Hinton agar according to CLSI guidelines [15]. E. coli ATCC25922 was used as quality control strain.

\section{Detection of quinolone resistance determinants (QRDs) and aminoglycoside resistance determinants (ARDs)} In this study, QRDs refer to plasmid mediated quinolone resistance genes. All the $75 \mathrm{CRE}$ were screened for the

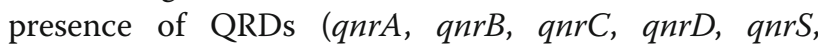
$a a c\left(6^{\prime}\right)-I b-c r, o q x A B$ and $q e p A$ ) and ARDs (aac(6')-Ib, $\operatorname{arm} A, n p m A, r m t A, r m t B, r m t C, r m t D$ and $r m t E)$ with PCR using primers as previously described [16, 17]. PCR products were purified and sequenced on an ABI PRISM 3730 automated sequencer (Applied Biosystems, Foster City, USA).

\section{Evaluation of concordance among methods}

The results were analyzed by using the standard agar dilution method as the reference method. Categorical agreement was defined as a result from any of the three automated system methods that belonged to the same interpretive category (i.e., susceptible, intermediate, or resistant) as that determined with the standard agar microdilution method. Essential agreement was defined as when the same MIC values (within \pm 1 dilution) were obtained by the automated systems and the reference method. In this study, when both MIC values obtained with automated systems and reference method were under or over the limit concentrations in the automated system, these results were considered to "agreement". A "very major error" was defined as a result in the susceptible category when an organism was considered resistant by the reference method, but susceptible by the test method. A "major error" was defined when an organism considered susceptible by the reference method was 
resistant by the test method. A "minor error" was defined when an organism was considered susceptible or resistant either by the reference or the test method, while intermediate by the other method. All tests showing very major errors or major errors were repeated in duplicate by both reference and test methods.

\section{Results}

\section{Prevalence of the QRDs and ARDs among CRE}

In total, 97.3\% (73/75) of CRE harbored QRDs. The qnr gene was the most common QRD determinant identified in 68 (96.7\%), followed by aac (6')-Ib-cr in 56 (74.7\%), $o q x A B$ in $23(30.7 \%)$, and $q e p A$ in $2(2.7 \%)$, respectively. Among the $68 q n r$ positive CRE, $q n r A, q n r B$, qnrS was detected in 64 (94.1\%), 17 (25.0\%), and 9 (13.2\%), respectively. $q n r C$ and $q n r D$ were not found in this study (Table 1).

22.7\% (17/75) of CRE harbored ARD determinants. $r m t A, r m t B$ and $n p m A$ were identified among these isolates in $6(8.0 \%), 6(8.0 \%)$ and $5(6.7 \%)$, respectively. $\mathrm{A} a c\left(6^{\prime}\right)-I b, \operatorname{arm} A, n p m A, \operatorname{rmt} C, r m t D$ and $r m t E$ were not detected in the present study.

\section{Resistance rates with reference agar dilution method}

Generally, based on the agar dilution method, the rates of resistance of CRE to CIP, LEV, GEN and AMK were 53.3\% (40/75), 37.3\% (28/75), 81.3\% (61/75) and 14.7\% (11/75), respectively (Table 2). The MIC ranges were $0.125-512 \mu \mathrm{g} / \mathrm{ml}$ for CIP, $0.125-128 \mu \mathrm{g} / \mathrm{ml}$ for LEV, $1-$ $512 \mu \mathrm{g} / \mathrm{ml}$ for GEN and $1-512 \mu \mathrm{g} / \mathrm{ml}$ for AMK. The $\mathrm{MIC}_{50}$ was $8 \mu \mathrm{g} / \mathrm{ml}$ for CIP, $2 \mu \mathrm{g} / \mathrm{ml}$ for LEV, $128 \mu \mathrm{g} / \mathrm{ml}$ for GEN and $8 \mu \mathrm{g} / \mathrm{ml}$ for AMK.

Performance on susceptibility of three automatic systems A total of 900 results were obtained in this study, corresponding to 300 for Phoenix, 300 for WalkAway and 300 for Vitek. Overall, the total error rate was 9.89\% (89/900).

Twenty-eight very major errors (3.11\%), 22 major errors $(2.44 \%)$ and 39 minor errors (4.33\%) were identified in this study against agar dilution method (Table 3 ). The very major errors were almost evenly distributed between results for fluoroquinolones $(2.89 \%)$ and aminoglycosides $(3.33 \%)$, while the major errors and minor errors were more commonly found in the results of quinolones $(3.11 \%$ and $6.44 \%$, respectively) than aminoglycosides ( $1.78 \%$ and $2.22 \%$, respectively). Very major errors were $3.67 \%$ of the total number of results for Vitek-2, 2.33\% for BD Phonix and 3.33\% for WalkAway. Major errors were $2.67 \%$ for Vitek-2, $2.00 \%$ for BD Phonix and $2.67 \%$ for WalkAway. Finally, minor errors were $6.00 \%$ for Vitek-2, $4.00 \%$ for BD Phonix and $3.00 \%$ for WalkAway. As to fluoroquinolones, most of the minor errors were susceptible findings interpreted as in intermediate, and resistance results interpreted as intermediate. The agreement in clinical categories was only
Table 1 Seventy five carbapenem-resistant Enterobacteriaceae isolates containing QDRs and ADRs that were detected in this study

\begin{tabular}{|c|c|c|}
\hline $\begin{array}{l}\text { Species } \\
\text { (no. of isolates) }\end{array}$ & $\begin{array}{l}\text { QDRs and } \\
\text { ADRs expressed }\end{array}$ & $\begin{array}{l}\text { No. of } \\
\text { isolates }\end{array}$ \\
\hline \multirow{8}{*}{$\begin{array}{l}\text { Escherichia } \\
\text { coli (18) }\end{array}$} & anrA & 2 \\
\hline & $q n r A+o q \times B$ & 1 \\
\hline & $a a c\left(6^{\prime}\right)-1 b-c r+a n r A$ & 6 \\
\hline & $a a c\left(6^{\prime}\right)-1 b-c r+a n r B$ & 1 \\
\hline & $a a c\left(6^{\prime}\right)-I b-c r+N P M A$ & 2 \\
\hline & $a a c\left(6^{\prime}\right)-l b-c r+a n r A+R m t B$ & 2 \\
\hline & $a a c\left(6^{\prime}\right)-1 b-c r+q n r A+N P M A$ & 3 \\
\hline & $o q \times B+q n r A$ & 1 \\
\hline \multirow{9}{*}{$\begin{array}{l}\text { Enterobacter } \\
\text { cloacae (20) }\end{array}$} & $a a c\left(6^{\prime}\right)-1 b-c r+q n r A+a n r B$ & 9 \\
\hline & $a a c\left(6^{\prime}\right)-1 b-c r+q n r A+q n r B+q n r S$ & 1 \\
\hline & $a a c\left(6^{\prime}\right)-1 b-c r+q n r B+a n r S$ & 1 \\
\hline & $a a c\left(6^{\prime}\right)-l b-c r+q n r A+o q \times B+o q \times A+R m t A$ & 1 \\
\hline & $a a c\left(6^{\prime}\right)-1 b-c r+q n r A$ & 2 \\
\hline & $a a c\left(6^{\prime}\right)-l b-c r+q n r A+q e p A$ & 1 \\
\hline & $a a c\left(6^{\prime}\right)-1 b-c r$ & 2 \\
\hline & qnrA & 2 \\
\hline & $a a c\left(6^{\prime}\right)-1 b-c r+q n r A+a n r S$ & 1 \\
\hline \multirow{19}{*}{$\begin{array}{l}\text { Klebsiella } \\
\text { pneumoniae (31) }\end{array}$} & qnrA & 3 \\
\hline & $q n r A+R m t B$ & 1 \\
\hline & $q n r A+R m t A$ & 2 \\
\hline & $q n r A+q n r B+o q \times A+o q \times B$ & 1 \\
\hline & $q n r A+o q x A+R m t A$ & 1 \\
\hline & $q n r A+q n r S+o q \times B$ & 1 \\
\hline & $q n r A+q n r S+o q \times A+o q \times B+R m t A$ & 1 \\
\hline & $a a c\left(6^{\prime}\right)-1 b-c r+a n r A$ & 1 \\
\hline & $a a c\left(6^{\prime}\right)-l b-c r+a n r A+o q x A$ & 1 \\
\hline & $a a c\left(6^{\prime}\right)-1 b-c r+a n r A+o q \times B$ & 3 \\
\hline & $a a c\left(6^{\prime}\right)-1 b-c r+a n r A+q e p A$ & 1 \\
\hline & $a a c\left(6^{\prime}\right)-1 b-c r+q n r A+q n r S+o q \times B$ & 1 \\
\hline & $a a c\left(6^{\prime}\right)-1 b-c r+q n r A+o q \times A+o q \times B$ & 8 \\
\hline & $a a c\left(6^{\prime}\right)-l b-c r+q n r A+q n r S+o q \times A+o q \times B$ & 1 \\
\hline & $a a c\left(6^{\prime}\right)-1 b-c r+a n r A+a n r B+o q \times B+R m t A$ & 1 \\
\hline & $\begin{array}{l}a a c\left(6^{\prime}\right)-\mid b-c r+q n r A+q n r B+q n r S \\
+o q \times B+o q \times A+R m t A\end{array}$ & 1 \\
\hline & $a a c\left(6^{\prime}\right)-1 b-c r+q n r A+o q \times B+o q \times A+N P M A$ & 1 \\
\hline & $\operatorname{aac}\left(6^{\prime}\right)-1 b-c r+o q \times B+o q \times A$ & 1 \\
\hline & oqxA+oqxB & 1 \\
\hline \multirow{2}{*}{$\begin{array}{l}\text { Klebsiella } \\
\text { oxytoca (4) }\end{array}$} & $a a c\left(6^{\prime}\right)-l b-c r+a n r A$ & 3 \\
\hline & $a a c\left(6^{\prime}\right)-1 b-c r+a n r B+a n r S$ & 1 \\
\hline $\begin{array}{l}\text { Enterobacter } \\
\text { aerogenes (1) }\end{array}$ & $a a c\left(6^{\prime}\right)-l b-c r+a n r B+a n r S$ & 1 \\
\hline
\end{tabular}


Table 2 Susceptibility of CRE based on testing methods in this study ( $N=75$ )

\begin{tabular}{|c|c|c|c|c|c|c|c|c|c|c|c|c|}
\hline \multirow{3}{*}{$\begin{array}{l}\text { Testing } \\
\text { method }^{a}\end{array}$} & \multicolumn{12}{|c|}{ Testing results (\%, [no. of strains]) } \\
\hline & \multicolumn{3}{|l|}{$\overline{C I P}$} & \multicolumn{3}{|l|}{ LEV } & \multicolumn{3}{|l|}{ GEN } & \multicolumn{3}{|l|}{ AMK } \\
\hline & $S$ & 1 & $\mathrm{R}$ & $\mathrm{s}$ & 1 & $\mathrm{R}$ & $\mathrm{s}$ & I & $\mathrm{R}$ & $S$ & 1 & $\mathrm{R}$ \\
\hline ADM & $37.3 \%(28)$ & $9.3 \%(7)$ & $53.3 \%(40)$ & $52.0 \%(39)$ & $10.7 \%(8)$ & $37.3 \%(28)$ & $17.3 \%(13)$ & $1.3 \%(1)$ & $81.3 \%(61)$ & $74.7 \%(56)$ & $10.7 \%(8)$ & $14.7 \%(11)$ \\
\hline Vitek-2 & $40.0 \%(30)$ & $8.0 \%(6)$ & $52.0 \%(39)$ & $52.0 \%(39)$ & $1.3 \%(1)$ & $46.7 \%(35)$ & $20.0 \%(15)$ & $2.7 \%(2)$ & $77.3 \%(58)$ & $86.7 \%(65)$ & 0 & $13.3 \%(10)$ \\
\hline Phoenix & $33.3 \%(25)$ & $8.0 \%(6)$ & $53.3 \%(40)$ & $42.7 \%(32)$ & $8.0 \%(6)$ & $48.0 \%(36)$ & $18.7 \%(14)$ & $4.0 \%(3)$ & $77.3 \%(58)$ & $84.0 \%(63)$ & $1.3 \%(1)$ & $14.7 \%(11)$ \\
\hline Microscan & $29.3 \%(22)$ & $8.0 \%(6)$ & $62.7 \%(47)$ & $45.3 \%(34)$ & $6.7 \%(5)$ & $48.0 \%(36)$ & $22.7 \%(17)$ & $4.0 \%$ (3) & $73.3 \%(55)$ & $80.0 \%(60)$ & $5.3 \%(4)$ & $14.7 \%(11)$ \\
\hline
\end{tabular}

${ }^{\mathrm{a} A D M}$, agar dilution method

76.0 to $85.4 \%$ for CIP and LEV. While as to aminoglycosides, most of the minor errors were resistance results interpreted as intermediate, or susceptible findings interpreted as in intermediate. The agreement in clinical categories was 86.7 to $92.0 \%$ for GEN and AMK.

\section{Discussion}

CRE have become to one of the most difficult-to-treat pathogens for nosocomial infections due to the lack of treatment options $[18,19]$. CRE are usually resistant to many commonly prescribed antimicrobials but may still remain susceptible to one or more antibiotics [20]. Aminoglycosides and fluoroquinolones are sometimes used as a choice to combat CRE infections currently [21, 22]. In China, automated systems are widely used nowadays not only in identification of bacteria, but to perform the antimicrobial susceptibility test as a routine in clinical microbiology laboratory. The present study was conducted to evaluate the performance of automated systems for the susceptibility testing on the two types of antibiotics (aminoglycosides and fluoroquinolones).

In this study, we found that QRD and ARD determinants were widely disseminated among CRE, which were similar to previous studies (Table 1) [23-26]. The resistance rates to fluoroquinolones were about 50\% (53.3\% to
CIP and $37.3 \%$ to LEV) based on the reference method, which was lower that in previous larger surveillance data from China [27]. The discrepancy probably due to the detection methods used in the previous study. In this study, the automated systems gave similar resistance rates to CIP, while the resistance rates to LEV were higher obtained with automated systems than that got from reference method. Meanwhile, the resistance rates to aminoglycosides vary with the specific drug $(81.7 \%$ to GEN and $14.7 \%$ to AMK) based on the reference method, which were in line with many previous studies $[24,27]$. In this study, the automated systems gave similar resistance rates to both GEN and AMK compared with the reference method.

Previous studies showed that automated systems were thought to be reliable for antibiotic susceptibility test, including aminoglycosides and fluoroquinolones [28, 29]. However, according to the US FDA's recommendation, the performance of susceptibility tests is considered adequate when the total error rate is $<10 \%$, with $\leq 1.5 \%$ of errors being very major errors and $\leq 3.0 \%$ being major errors, and when the overall essential MIC agreement is $>90 \%$ [30]. Taking these values as a reference, the three automated systems cannot be considered reliable for susceptibility testing of aminoglycosides and

Table 3 Summary of the results on CRE obtained with automated methods compared to the results with reference method

\begin{tabular}{|c|c|c|c|c|c|c|}
\hline \multirow[t]{2}{*}{ Antibiotics } & \multirow[t]{2}{*}{ System (concns in mg/l) } & \multirow[t]{2}{*}{$\%$ agreement in clinical categories } & \multirow[t]{2}{*}{$\%$ essential agreement } & \multicolumn{3}{|c|}{ No. of errors of different types (\%) } \\
\hline & & & & Very major & Major & Minor \\
\hline \multirow[t]{3}{*}{$\mathrm{CIP}$} & WalkAway $(0.12,1,2)$ & 76.0 & 81.3 & $2(0.2)$ & $4(0.4)$ & $4(0.4)$ \\
\hline & BD Phoenix $(0.12,0.25,0.5,1,2)$ & 80.0 & 88.0 & $2(0.2)$ & $1(0.1)$ & $4(0.4)$ \\
\hline & Vitek-2 $(0.25,0.5,1,2,4)$ & 85.4 & 84.0 & $3(0.3)$ & $2(0.2)$ & $7(0.8)$ \\
\hline \multirow[t]{3}{*}{ LEV } & Walkaway $(4,8)$ & 78.7 & 86.7 & $2(0.2)$ & $2(0.2)$ & $3(0.3)$ \\
\hline & BD Phoenix $(2,4,8)$ & 77.3 & 84.0 & $1(0.1)$ & $2(0.2)$ & $4(0.4)$ \\
\hline & Vitek-2 $(1,2,4,8,16)$ & 81.3 & 81.3 & $3(0.3)$ & $3(0.3)$ & $7(0.8)$ \\
\hline \multirow[t]{3}{*}{ GEN } & Walkaway $(4,8)$ & 86.7 & 89.3 & $5(0.6)$ & $1(0.1)$ & $2(0.2)$ \\
\hline & BD Phoenix $(2,4,8)$ & 90.7 & 90.7 & $3(0.3)$ & $2(0.2)$ & $3(0.3)$ \\
\hline & Vitek-2 $(1,2,4,8,16)$ & 89.3 & 90.7 & $3(0.3)$ & $2(0.2)$ & $3(0.3)$ \\
\hline \multirow[t]{3}{*}{ AMK } & Walkaway $(8,16,32)$ & 92.0 & 94.7 & $1(0.1)$ & $1(0.1)$ & 0 \\
\hline & BD Phoenix $(8,16,32)$ & 90.7 & 89.3 & $1(0.1)$ & $1(0.1)$ & $1(0.1)$ \\
\hline & Vitek-2 $(8,16,32)$ & 88.0 & 84.0 & $2(0.2)$ & $1(0.1)$ & $1(0.1)$ \\
\hline
\end{tabular}


fluoroquinolones against CRE in our study for the high very major error rate and overall essential MIC agreement (Table 3).

Our study has several limitations. The study was carried out at a single research laboratory. To reduce biases, the measurements were performed in biological duplicates by blinded researchers on different days. Another limitation was the relatively small sample size. For this reason, we were unable to obtain comprehensive evaluation of the three automated systems for aminoglycoside and fluoroquinolones susceptibility testing for CRE. Lastly, only one reference method was chosen in this study. More accurate methods (such as broth dilution method, E-test) should be used to confirm the AST results.

\section{Conclusions}

This is the first study assessing the accuracy of automated systems in detecting the susceptibility of aminoglycosides and fluoroquinolones to CRE. Our limited study suggests that testing difficulties in susceptibility testing do exist in automated systems. These findings warn us that reporting errors could result in serious implications for the clinical outcome for patients. We suggest clinical laboratories using automated systems should consider using a second, independent antimicrobial susceptibility testing method to validate aminoglycosides and fluoroquinolones susceptibility.

\section{Abbreviations}

ARDs: Aminoglycoside resistance determinant; CRE: Carbapenem resistant Enterobacteriaceae; MIC: Minimum inhibitory concentration; QRDs: Quinolone resistance determinants

\section{Acknowledgements}

Not applicable.

\section{Funding}

This study was supported by the Fujian Provincial Funds for Distinguished Young Scientists in Colleges and Universities, China (grant no. JA13134), and the Medical Elite Cultivation Program of Fujian, China (grant no. 2015-ZQN-ZD-15).

\section{Availability of data and materials}

All the data supporting the findings are presented in the manuscript.

\section{Authors' contributions}

BL designed the experiment and wrote the manuscript. ZZ, WC, LH and QL performed the experiments. FL and ML participated in data analysis and review of the draft manuscript. All authors read and approved the final manuscript.

\section{Ethics approval and consent to participate}

Not applicable.

\section{Consent for publication}

Not applicable.

\section{Competing interests}

The authors declare that they have no competing interests.

\section{Publisher's Note}

Springer Nature remains neutral with regard to jurisdictional claims in published maps and institutional affiliations.

\section{Author details}

'Department of Pharmacy, Fujian Medical University Union Hospital, Fuzhou, Fujian 350001, China. ${ }^{2}$ Department of Clinical Laboratory, Fujian Medical University Union Hospital, 29 Xinquan Rd, Fuzhou, Fujian 350001, People's Republic of China. ${ }^{3}$ Medical Technology and Engineering College, Fujian Medical University, Fuzhou 350004, Fujian, People's Republic of China.

Received: 19 June 2017 Accepted: 25 July 2017

Published online: 01 August 2017

\section{References}

1. Chea N, Bulens SN, Kongphet-Tran T, Lynfield R, Shaw KM, Vagnone PS, et al. Improved phenotype-based definition for identifying Carbapenemase producers among Carbapenem-resistant Enterobacteriaceae. Emerg Infect Dis. 2015:21:1611-6.

2. Potter RF, D'Souza AW, Dantas G. The rapid spread of carbapenem-resistant Enterobacteriaceae. Drug Resist Updat. 2016;29:30-46.

3. Logan LK, Weinstein RA. The epidemiology of Carbapenem-resistant Enterobacteriaceae: the impact and evolution of a global menace. J Infect Dis. 2017;215:S28-36.

4. Esterly JS, Wagner J, McLaughlin MM, Postelnick MJ, Qi C, Scheetz MH. Evaluation of clinical outcomes in patients with bloodstream infections due to gram-negative bacteria according to carbapenem MIC stratification. Antimicrob Agents Chemother. 2012;56:4885-90.

5. Skurnik D, Roux D, Pons S, Guillard T, Lu X, Cywes-Bentley C, et al. Extendedspectrum antibodies protective against carbapenemase-producing Enterobacteriaceae. J Antimicrob Chemother. 2016;71:927-35.

6. Friedman ND, Carmeli Y, Walton AL, Schwaber MJ. Carbapenem-resistant Enterobacteriaceae: a strategic roadmap for infection control. Infect Control Hosp Epidemiol. 2017;38:580-94.

7. Morrill HJ, Pogue JM, Kaye KS, KL LP. Treatment options for Carbapenemresistant Enterobacteriaceae infections. Open Forum Infect Dis. 2015;2: ofv050.

8. Ni W, Wei C, Zhou C, Zhao J, Liang B, Cui J, Wang R, et al. TigecyclineAmikacin combination effectively suppresses the selection of resistance in clinical isolates of KPC-producing Klebsiella Pneumoniae. Front Microbiol. 2016:7:1304.

9. van Duin D, Kaye KS, Neuner EA, Bonomo RA. Carbapenem-resistant Enterobacteriaceae: a review of treatment and outcomes. Diagn Microbiol Infect Dis. 2013;75:115-20.

10. Perez F, El Chakhtoura NG, Papp-Wallace KM, Wilson BM, Bonomo RA. Treatment options for infections caused by carbapenem-resistant Enterobacteriaceae: can we apply "precision medicine" to antimicrobial chemotherapy? Expert Opin Pharmacother. 2016:17:761-81.

11. Hsu AJ, Tamma PD. Treatment of multidrug-resistant gram-negative infections in children. Clin Infect Dis. 2014;58:1439-48.

12. Paterson DL. Impact of antibiotic resistance in gram-negative bacilli on empirical and definitive antibiotic therapy. Clin Infect Dis. 2008;47:S14-20.

13. Hu FP, Guo Y, Zhu DM, Wang F, Jiang XF, Xu YC, et al. Resistance trends among clinical isolates in China reported from CHINET surveillance of bacterial resistance, 2005-2014. Clin Microbiol Infect. 2016;22(Suppl 1):S9-14.

14. He Q, Chen W, Huang L, Lin Q, Zhang J, Liu R, et al. Performance evaluation of three automated identification systems in detecting carbapenemresistant Enterobacteriaceae. Ann Clin Microbiol Antimicrob. 2016;15:40.

15. CLSI. Performance Standards for Antimicrobial Susceptibility Testing. 27th ed. CLSI supplement M100. Wayne, PA: Clinical and Laboratory Standards Institute; 2017.

16. Doi Y, Arakawa Y. 165 ribosomal RNA methylation: emerging resistance mechanism against aminoglycosides. Clin Infect Dis. 2007:45:88-94.

17. Chen X, Zhang W, Pan W, Yin J, Pan Z, Gao S, et al. Prevalence of qnr, aac(6)$\mathrm{lb}-\mathrm{cr}$, qepA, and oqxAB in Escherichia Coli isolates from humans, animals, and the environment. Antimicrob Agents Chemother. 2012;56:3423-7.

18. Wang Q, Zhang Y, Yao X, Xian H, Liu Y, Li H, et al. Risk factors and clinical outcomes for carbapenem-resistant Enterobacteriaceae nosocomial infections. Eur J Clin Microbiol Infect Dis. 2016;35:1679-89.

19. Vergara-Lopez S, Dominguez MC, Conejo MC, Pascual A, Rodriguez-Bano J. Lessons from an outbreak of metallo-beta-lactamase-producing Klebsiella oxytoca in an intensive care unit: the importance of time at risk and combination therapy. J Hosp Infect. 2015;89:123-31.

20. Trecarichi EM, Tumbarello M. Therapeutic options for carbapenem-resistant Enterobacteriaceae infections. Virulence. 2017;8:470-84. 
21. Lesho EP, Clifford RJ, Chukwuma U, Kwak YI, Maneval M, Neumann C, et al. Carbapenem-resistant Enterobacteriaceae and the correlation between carbapenem and fluoroquinolone usage and resistance in the US military health system. Diagn Microbiol Infect Dis. 2015;81:119-25.

22. Zavascki AP, Klee BO, Bulitta JB. Aminoglycosides against carbapenemresistant Enterobacteriaceae in the critically ill: the pitfalls of aminoglycoside susceptibility. Expert Rev Anti-Infect Ther. 2017;15:519-26.

23. Dai W, Sun S, Yang P, Huang S, Zhang X, Zhang L. Characterization of carbapenemases, extended spectrum beta-lactamases and molecular epidemiology of carbapenem-non-susceptible Enterobacter Cloacae in a Chinese hospital in Chongqing. Infect Genet Evol. 2013;14:1-7.

24. Hu L, Zhong Q, Shang Y, Wang H, Ning C, Li Y, et al. The prevalence of carbapenemase genes and plasmid-mediated quinolone resistance determinants in carbapenem-resistant Enterobacteriaceae from five teaching hospitals in central China. Epidemiol Infect. 2014;142:1972-7.

25. Rahman M, Shukla SK, Prasad KN, Ovejero CM, Pati BK, Tripathi A, et al. Prevalence and molecular characterisation of New Delhi metallo-betalactamases NDM-1, NDM-5, NDM-6 and NDM-7 in multidrug-resistant Enterobacteriaceae from India. Int J Antimicrob Agents. 2014;44:30-7.

26. Cheng L, Cao XL, Zhang ZF, Ning MZ, Xu XJ, Zhou W, et al. Clonal dissemination of KPC-2 producing Klebsiella Pneumoniae ST11 clone with high prevalence of oqx $A B$ and rmtB in a tertiary hospital in China: results from a 3-year period. Ann Clin Microbiol Antimicrob. 2016;15:1.

27. Xu A, Zheng B, Xu YC, Huang ZG, Zhong NS, Zhuo C. National epidemiology of carbapenem-resistant and extensively drug-resistant gram-negative bacteria isolated from blood samples in China in 2013. Clin Microbiol Infect. 2016;22:S1-8.

28. Snyder JW, Munier GK, Johnson CL. Direct comparison of the BD phoenix system with the MicroScan WalkAway system for identification and antimicrobial susceptibility testing of Enterobacteriaceae and nonfermentative gram-negative organisms. J Clin Microbiol. 2008:46:2327-33.

29. Calvo J, Cano ME, Pitart C, Marco F, Rodriguez-Martinez JM, Pascual A, et al. Evaluation of three automated systems for susceptibility testing of enterobacteria containing qnrB, qnrS, and/or aac(6')-lb-cr. J Clin Microbiol. 2011:49:3343-5.

30. U.S.Food and Drug Administration. Class II specail controls guidance document antimicrobial susceptibility test (AST) systems; quidance for industry and FDA. Rockville. MD: U.S. Food and Drug Administration; 2007.

\section{Submit your next manuscript to BioMed Central and we will help you at every step:}

- We accept pre-submission inquiries

- Our selector tool helps you to find the most relevant journal

- We provide round the clock customer support

- Convenient online submission

- Thorough peer review

- Inclusion in PubMed and all major indexing services

- Maximum visibility for your research

Submit your manuscript at www.biomedcentral.com/submit

) Biomed Central 